\title{
ON CERTAIN SEQUENCES DERIVED FROM GENERALIZED EULER-MASCHERONI CONSTANTS
}

\section{TIBERIU TRIF}

Abstract. Let $0<\alpha<1$, and let

$$
C_{\alpha}:=\lim _{n \rightarrow \infty}\left(1+\frac{1}{2^{\alpha}}+\cdots+\frac{1}{n^{\alpha}}-\frac{n^{1-\alpha}}{1-\alpha}\right) .
$$

It is proved that there exists a unique sequence $\left(\omega_{n}\right)$ such that

$$
1+\frac{1}{2^{\alpha}}+\cdots+\frac{1}{n^{\alpha}}=C_{\alpha}+\frac{\left(n+\omega_{n}\right)^{1-\alpha}}{1-\alpha} .
$$

Moreover, the sequence $\left(\omega_{n}\right)$ is decreasing and satisfies $\frac{1}{2} \leqslant \omega_{n} \leqslant \frac{1}{4}\left[1+\left(1+\frac{1}{n}\right)^{\alpha}\right]$, whence $\lim _{n \rightarrow \infty} \omega_{n}=\frac{1}{2}$. This is only a special case of the more general results established in this paper. These results concern some sequences derived from generalized Euler-Mascheroni constants involving convex functions and complement similar ones obtained by V. Timofte [Integral estimates for convergent positive series. J. Math. Anal. Appl. 303 (2005), 90-102].

Mathematics subject classification (2010): 11B83, 26D15.

Keywords and phrases: Euler-Mascheroni constant, convex function, Hermite-Hadamard inequality.

\section{REFERENCES}

[1] W. W. Breckner And T. TRIF, Convex Functions and Related Functional Equations. Selected Topics, Cluj University Press, Cluj-Napoca, 2008.

[2] D. W. DeTemple, A quicker convergence to Euler's constant, Amer. Math. Monthly, 100 (1993), 468-470.

[3] G. H. Hardy, J. E. Littlewood and G. Pólya, Inequalities, Cambridge University Press, 1934.

[4] C. P. Niculescu And L.-E. Persson, Convex Functions and Their Applications. A Contemporary Approach, Springer-Verlag, New York, 2006.

[5] A. W. Roberts and D. E. Varberg, Convex Functions, Academic Press, New York, London, 1973.

[6] J. SÁNDOR, On generalized Euler constants and Schlömilch-Lemonnier type inequalities, J. Math. Anal. Appl., 328 (2007), 1336-1342.

[7] V. Timofte, Integral estimates for convergent positive series, J. Math. Anal. Appl., 303 (2005), 90102.

[8] L. Toth, Problem E3432, Amer. Math. Monthly, 98 (1991), 264.

[9] L. Toth, Problem E3432 (Solution), Amer. Math. Monthly, 99 (1992), 684-685. 